En 1996, le Forum du sauvetage exigeait déjà dans ses «12 thèses sur le domaine du sauvetage en Suisse» que «le numéro d'urgence sanitaire 144 devienne le point de contact gratuit et uniformément valable en Suisse pour toutes les situations d'urgence sanitaire». Grâce aux centrales d'appels sanitaires d'urgence, le numéro 144 garantit le tri nécessaire des appels et le bon déroulement du sauvetage visant ainsi une prise en charge rapide et adaptée des patients en situation d'urgence.

Dr Ernst Gähler, vice-président de la FMH, responsable du département Tarifs et conventions pour la médecine ambulatoire en Suisse

\title{
Les centrales d'appels sanitaires d'urgence - pivot capital du sauvetage en Suisse
}

\author{
Wolfgang Ummenhofer ${ }^{a}$, \\ Stefan Müller ${ }^{b}$, \\ Günter Bildstein
}

a Professeur, service d'anesthésie de l'Hôpital universitaire de Bâle

b Dr, médecin-chef, protection et sauvetage, Zurich

c Günter Bildstein, MSc, MBA, Président de la Commission CASU 144 de l'IAS, sauvetage de Saint-Gall
Correspondance: Prof. Wolfgang Ummenhofer Service d'anesthésie Hôpital universitaire de Bâle CH-4031 Bâle
En 1996, le Forum du sauvetage exigeait déjà dans ses «12 thèses sur le domaine du sauvetage en Suisse» que «le numéro d'urgence sanitaire 144 devienne le point de contact gratuit et uniformément valable en Suisse pour toutes les situations d'urgence sanitaire». Entre-temps, 18 centrales d'appels sanitaires d'urgence (CASU) coordonnent pas moins de 450000 interventions annuelles pour 115 services de sauvetage à quelque 160 points de contact. Toutes en lien les unes avec les autres, elles reçoivent 600000 appels environ, directement via le numéro d'urgence 144 - tendance à la hausse. Du point de vue économique, on considère qu'une centrale d'appels sanitaires d'urgence atteint une «dimension raisonnable» lorsqu'elle dessert une région d'au moins 500000 habitants; dans notre pays, ce seuil n'est pas toujours atteint, principalement pour des raisons politiques.

Dans la plupart des cas, les appels sont gérés par des ambulanciers diplômés avec une formation CASU spécifique alors que la direction médicale est assurée par un médecin urgentiste SSMUS. Les CASU «classiques» gèrent exclusivement les appels au 144; la conjonction de centrales d'appels sanitaires d'urgence et de centrales de pompiers (118) existent au même titre que le regroupement des appels d'urgence pour médecins, pompiers et policiers (117) dans une seule et même centrale. Il est évident que ces regroupements peuvent s'avérer judicieux du point de vue technique et organisationnel mais rien ne confirme que de telles «centrales intégrées» permettent de créer d'importantes synergies ou de réduire le personnel. D'une part, les appels concernant une urgence médicale doivent être triés par des personnes spécialement formées à cet effet, et d'autre part, le nombre d'appels traités par les centrales intégrées augmente de manière significative et proportionnelle à l'extension du service proposé. Comme dans d'autres pays européens, les politiciens ont exigé de la Suisse de mettre en place un seul numéro d'urgence à trois chiffres pour toutes les situations d'urgence (112). Les expériences acquises en Finlande après un tel fusionnement ont cependant montré une hausse du temps consacré aux appels et une augmentation du nombre total d'interventions avec ambulance. Dans l'ensemble, peu d'effets positifs ont été enregistrés et le recours à des moyens de sauvetage inadéquats a augmenté [1].

Les CASU trient tous les appels d'urgence et organisent de façon géographiquement appropriée les premiers secours. Selon la situation

- la personne qui demande de l'aide recevra des instructions concrètes afin d'attendre dans les meilleures conditions l'arrivée des équipes de secours, - le patient en détresse sera renvoyé au médecin de garde ou à un cabinet d'urgence,

- l'intervention de l'ambulance la plus proche et, en cas d'indication médicale, du médecin d'urgence ou éventuellement du médecin de garde sera déclenchée,

- l'intervention de l'hélicoptère de secours sera déclenchée en cas d'indication médicale ou pour le bon déroulement des secours (par ex. longue voie d'accès ou absence de chemin d'accès), ce qui permet d'envoyer un médecin d'urgence pour des événements spécifiques (par ex. en cas d'urgence pédiatrique grave) mais aussi d'assurer un transport rapide vers un hôpital approprié,

- le recours à l'ambulancier chef des secours (ACS) et au médecin-chef des secours (MCS) est déclenché, - la CASU sert de dispositif de mobilisation des services sanitaires (backoffice) en cas d'événement grave et de catastrophe.

Dans son rôle de forum des sociétés de discipline, le Forum du sauvetage de la FMH a élaboré des lignes directrices pour une assistance préhospitalière d'urgence uniforme et obligatoire, également en cas d'événements de grande ampleur. La FMH s'engage pour que les soins urgents soient assurés par les urgentistes, ou des généralistes spécialement formés, et les ambulanciers. En été 2010, le Forum du sauvetage a actualisé ses thèses publiées en 1996 (Bulletin des médecins suisses $n^{\circ} 33,2010$ et www.fmh.ch $\rightarrow$ services $\rightarrow$ Forum du sauvetage). Le BMS consacrera une série d'articles périodiques qui traiteront plus en détail les huit points énoncés dans les nouvelles lignes directrices. 
Parce qu'elles sont structurées et standardisées, les procédures employées pour répondre aux appels d'urgence améliorent le tri des cas grâce notamment à des logiciels et à des algorithmes spécifiques. Elles aident également à déclencher le sauvetage adapté à la situation du patient ou à suivre la structure souhaitée avec des soins médicaux appropriés. Par ailleurs, les mesures immédiates transmises par téléphone aux proches ou aux premiers secouristes, lors d'une intervention d'urgence sur un patient dont le pronostic vital est engagé, prennent de plus en plus d'importance. Celles-ci doivent être communiquées de manière adéquate en tenant compte de la situation émotionnelle exceptionnelle. Définis sous la houlette du responsable médical de la CASU, les scénarios possibles (par ex. les instructions pour un massage cardiaque) doivent être régulièrement examinés et vérifiés, et conservés de manière plausible pour les procédures de certification. Tout cela peut paraître simple mais requiert un apprentissage intensif et un entraînement régulier pour que les instructions fonctionnent dans des conditions difficiles (en cas de réanimation par exemple), qu'elles soient compréhensibles au téléphone par des profanes et que ceux-ci puissent les mettre en œuvre de manière efficace.

Les employés d'une CASU doivent maîtriser les compétences médicales de base. Dans l'idéal, ils disposent d'un diplôme d'ambulancier ES et d'une expérience régulière dans les interventions de sauvetage. Aujourd'hui, la plupart des responsables de CASU procèdent eux-mêmes à la formation à l'interne de leurs ambulanciers chargés de la gestion des interventions; à la demande des organisations concernées, une commission développe actuellement un profil de formation spécifique à la gestion des appels. Les contenus et aspects techniques, logistiques et psychologiques entrant en ligne de compte dans la manière de gérer les appels d'urgence doivent être traités selon un système de modules afin de pouvoir être également utiles et applicables aux équipes de la police et des pompiers.

Le transfert vers un cabinet de garde en dehors des heures d'ouverture habituelle est en partie aussi du ressort des centrales d'appels sanitaires d'urgence. Mais le plus souvent, ce sont des centres d'appels, comme la "Centrale des médecins», qui se chargent de diriger les patients. Il est cependant essentiel de séparer nettement les urgences graves nécessitant un sauvetage d'un transfert vers un cabinet médical en dehors des heures d'ouverture. A cet égard, la communication requise à plusieurs niveaux ne doit pas être sous-estimée. Il peut arriver qu'une ambulance intervienne auprès d'un patient qui ne souhaitait que la visite à domicile d'un médecin urgentiste mais aussi, heureusement beaucoup plus rarement, qu'un patient gravement malade doive attendre longtemps avant une prise en charge invasive sur place et son transfert d'urgence à l'hôpital.

Au-delà des moyens technologiques de plus en plus sophistiqués, nous devons davantage veiller à la formation des organisations partenaires et des équipes qui constituent les centrales d'appels d'urgence. Communication, Crew Resource et Task Management, anticipation des éventuelles perspectives professionnelles, évaluation des risques et choix parmi les différentes options sont des concepts de plus en plus importants. Ce d'autant plus que les CASU deviennent un pivot décisif pour alarmer les responsables des secours et le personnel intervenant lors d'évènements extraordinaires. En tant que centre de commandement des ambulanciers, elles permettent aux responsables d'intervenir sur place selon un plan d'action médical structuré. Mais pour augmenter leur efficacité, les CASU existantes doivent fusionner et former des unités plus importantes dont le périmètre d'intervention devrait s'orienter sur des régions géographiques plutôt que nécessairement sur des frontières cantonales.

\section{Principes fondamentaux}

- L'organisation appropriée et efficace des urgences médicales repose sur des centrales d'appels sanitaires d'urgence suprarégionales dont la dimension dépasse le seuil critique.

- Un numéro d'urgence unique et gratuit existe déjà (CASU 144) - un numéro d'urgence commun comme le 112 (police, pompiers, ambulanciers), indépendant de l'événement, ne présente aucun avantage.

- En plus du tri des cas d'une part et de la direction de l'intervention des secouristes d'autre part, il est nécessaire de créer des instructions téléphoniques pour les proches de patients en détresse ou pour les premiers secouristes.

- La formation à la «gestion des interventions» est en cours d'élaboration et doit être réglementée au niveau national.

- L'interface entre CASU 144 et les centrales téléphoniques des organisations de médecins doit être définie et organisée en conséquence en ce qui concerne le service de piquet des médecins de garde et des services d'urgences du secteur ambulatoire. Possibilités de synergies en matière de personnel et de logistique.

- L'expérience montre qu'on accorde plus d'importance aux investissements technologiques qu'à ceux liés au personnel. Cependant, en matière de communication et de direction, il faut former davantage tous les responsables CASU, à savoir celui qui prend les appels, celui qui gère les interventions, le responsable médical et le responsable des secours.

- Cette formation revêt une importance particulière en vue de les préparer à diriger des interventions en situation particulière ou extraordinaire.

* Nous remercions Heinz Zimmermann pour sa relecture critique du manuscrit.

1 Määttä T, et al. Fusion of dispatching centres into one entity: effects on performance. Acta Anaesthesiol Scand. 2010;54:689-95. 